ARTICLE

https://doi.org/10.1038/s41467-019-12520-y

\title{
Contribution of retrotransposition to developmental disorders
}

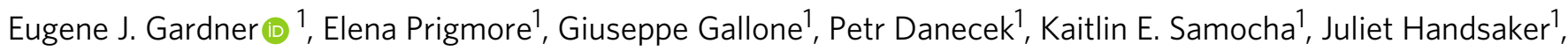
Sebastian S. Gerety (1) 1, Holly Ironfield ${ }^{1}$, Patrick J. Short (1) ${ }^{1}$, Alejandro Sifrim², Tarjinder Singh (1) ${ }^{1}$, Kate E. Chandler ${ }^{3}$, Emma Clement ${ }^{4}$, Katherine L. Lachlan ${ }^{5,6}$, Katrina Prescott ${ }^{7}$, Elisabeth Rosser ${ }^{4}$, David R. FitzPatrick ${ }^{8}$, Helen V. Firth ${ }^{1,9}$ \& Matthew E. Hurles ${ }^{1 *}$

Mobile genetic Elements (MEs) are segments of DNA which can copy themselves and other transcribed sequences through the process of retrotransposition (RT). In humans several disorders have been attributed to RT, but the role of RT in severe developmental disorders (DD) has not yet been explored. Here we identify RT-derived events in 9738 exome sequenced trios with DD-affected probands. We ascertain 9 de novo MEs, 4 of which are likely causative of the patient's symptoms (0.04\%), as well as 2 de novo gene retroduplications. Beyond identifying likely diagnostic RT events, we estimate genome-wide germline ME mutation rate and selective constraint and demonstrate that coding RT events have signatures of purifying selection equivalent to those of truncating mutations. Overall, our analysis represents a comprehensive interrogation of the impact of retrotransposition on protein coding genes and a framework for future evolutionary and disease studies.

\footnotetext{
${ }^{1}$ Wellcome Sanger Institute, Wellcome Genome Campus, Cambridge, Hinxton CB10 1SA, UK. ${ }^{2}$ Department of Human Genetics, KU Leuven, Herestraat 49 Box 602 Leuven B-3000, Belgium. ${ }^{3}$ Manchester Centre for Genomic Medicine, Manchester University Hospitals NHS Foundation Trust, Manchester Academic Health Science Centre, Manchester, Greater, Manchester M13 9WL, UK. ${ }^{4}$ Department of Clinical Genetics, North East Thames Regional Genetics Service, Great Ormond Street Hospital for Children NHS Trust, Holborn, London WC1N 3JH, UK. ${ }^{5}$ Wessex Clinical Genetics Service, Southampton University Hospitals NHS Foundation Trust, Princess Anne Hospital, Southampton SO16 5YA, UK. ${ }^{6}$ Faculty of Medicine, Human Development and Health, University of Southampton, Southampton SO17 1BJ, UK. 7 Clinical Genetics Department, Yorkshire Regional Genetics Service, Leeds Teaching Hospitals NHS Trust, Chapel Allerton Hospital, Leeds LS7 4SA, UK. ${ }^{8}$ MRC Human Genetics Unit, MRC IGMM, University of Edinburgh, WGH, Edinburgh EH4 2SP, UK. 9 East Anglian Medical Genetics Service, Box 134, Cambridge University Hospitals NHS Foundation Trust, Cambridge Biomedical Campus, Cambridge CB2 OQQ, UK.

*email: meh@sanger.ac.uk
} 
n humans, three classes of mobile genetic elements (MEs)$A l u$, long interspersed nuclear element 1 (L1), and SINEVNTR-Alu (SVA)-are still active and can generate new copies, known as mobile element insertions (MEIs), throughout their host genome ${ }^{1}$. The $\mathrm{L} 1$ replicative machinery can also facilitate the duplication of non-ME transcripts, typically proteincoding genes, through the mechanism of retroduplication to generate processed pseudogenes (PPGs) ${ }^{2}$. Combined, these two processes constitute retrotransposition (RT) in the human genome, with new (de novo) MEI variants previously estimated to occur in every 1 out of $18.4-26.0$ births $^{3}$. On a population level, each individual human genome harbors $\sim 1200$ polymorphic variants, with the smallest $\mathrm{ME}, A l u$, generally contributing $75 \%$ of total RT polymorphisms ${ }^{4-6}$.

To date, roughly 130 pathogenic variants caused by RT activity have been documented ${ }^{7}$; however, the majority of these deleterious events have been discovered in isolated cases. Neither MEIs nor PPGs are canalyzed as part of routine clinical sequencing, and thus represent a largely unassessed category of genetic variation in many disorders. Furthermore, of the clinically relevant RTattributable cases thus identified, few $(\sim 14 / 123 ; 11.4 \%)$ are caused by new mutational events and are instead typically attributable to rare inherited polymorphisms ${ }^{7}$. In addition, of the large diseasefocused whole-genome sequencing (WGS) projects which have ascertained MEIs, all have focused on autism ${ }^{8-10}$ and have failed to identify likely causative RT-derived variants. In fact, in the largest and most recent WGS study investigating the role of large structural variants in the genetic architecture of autism, the authors failed to identify a single de novo MEI in a coding exon, deleterious or otherwise, in 829 families $^{9}$. This finding is likely a result of several factors, predominant among them the low frequency of cases attributable to gene disruption by MEIs in autism ${ }^{10}$, due in part to a low ME mutation rate $^{3}$ and lack of a sufficiently large sample size $e^{8,9,11}$. As such, it is not precisely known at what rate de novo ME variants are generated in the human genome, the functional consequences of such variants, the role that they play in the etiology of rare disease, and if routine clinical sequencing should assess patient genomes for deleterious RT events.

Herein, we analyze the whole-exome sequencing (WES) data produced by the Deciphering Developmental Disorders (DDD) study to systematically assess the role of RT in severe developmental disorders (DDs). The DDD data have already been investigated for pathogenic single-nucleotide variants (SNVs), small insertions and deletions (InDels), large copy number variants $(\mathrm{CNVs})$, and other classes of variation ${ }^{12-18}$. Approximately, $24 \%$ of DDD cases harbor a pathogenic de novo mutation in a gene known to be associated with developmental disorders ${ }^{12}$. The DDD cohort should thus be relatively enriched for highly penetrant de novo RT events in comparison with recent studies on autism ${ }^{8,9}$. With a cohort of 9738 trios ( $n=28,132$ individuals) whole-exome sequenced, the DDD study presents a powerful opportunity to identify, and ascertain the role in DD of, pathogenic de novo RT events that impact coding sequences.

\section{Results}

Generation of a genome-wide data set of RT variants. To assess 9738 DDD study trios for RT events, we utilized two separate computational approaches to identify both MEIs and PPGs. First, we used the mobile element locator tool (MELT) ${ }^{5}$ to identify Alu, L1, and SVA variants located within the WES bait regions (see the Methods section). The second is a new bespoke tool developed to identify PPGs from the WES data (Methods, Supplementary Fig. 1). Due to cross-hybridization between a PPG and the exome baits targeting the donor gene, we anticipated that we should be able to detect PPGs genome wide, not just the subset that insert within the WES bait regions. Our PPG detection tool ascertained putative PPGs by identifying multiple discordant read pairs mapping to different exons of the same transcript, before then typing all individuals for the presence/absence of the PPG using discordant read pairs and split reads. The tool was optimized by comparing against previously described PPG polymorphisms in the 1000 genomes project (1KGP; see below).

As our study is the first to discover MEIs directly from WES on a large scale with MELT, we first utilized matched sample WGS data to determine if MELT could ascertain MEI variants reliably from the WES data. We compared MEI variants identified by MELT within the DDD WES data to both WGS data generated for 90 overlapping DDD individuals and population MEI data previously generated from the 1000 Genomes Project Phase 3 (1KPG) $)^{4-6}$ WGS data. The latter comparison was to ensure that the number of exonic MEIs identified within DDD WES data was concordant with expectations at the individual and population level. When comparing our WES genotypes to WGS in the same individuals, we had a genotype concordance rate of $94.46 \%$ (93.93\% Alu, 97.29\% L1, 98.25\% SVA) among calls with at least $10 \times$ coverage in our WES data. In total, we were able to reidentify 1450 (1289 Alu, 160 L1, 1 SVA) MEI genotypes, or 84.5\% of all heterozygous or homozygous genotypes identifiable with WGS in WES bait regions (Supplementary Table 1). Based on these findings, we were confident that MELT was appropriately calibrated to ascertain MEIs in WES data.

We identified 1129 MEI variants and 576 polymorphic PPGs, with each individual's exome containing on average $33.0 \pm 5.0$ (SD) variants (Table 1). All MEIs were genotyped across all individuals to form a comprehensive catalogue of RT-derived variation within and adjacent to $( \pm 50 \mathrm{bp})$ sequences targeted in the WES assay (Methods), including coding exons and targeted noncoding elements (Table 1; Fig. 1). The average time to assess a single family for RT-derived events was $\sim 15 \mathrm{~min}$, and the rate of false findings was low (1 incorrect candidate de novo variant per every 295 patients; either a false-positive variant [1 per 649] or falsenegative genotype [1 per 541] in at least one parent). We investigated whether false-negative parental genotypes could be

Table 1 RT variant discovery in the DDD study

\begin{tabular}{llc} 
& Total sites cohort-wide & Mean sites per unaffected parent \\
\hline Alu & 917 & $23.6 \pm 4.2$ \\
LINE-1 & 167 & $2.8 \pm 1.5$ \\
SVA & 45 & $0.2 \pm 0.5$ \\
Total - MEI & 1129 & $26.6 \pm 4.7$ \\
Processed pseudogenes (PPGs) & 576 & $6.6 \pm 1.7$ \\
Total - MEI + PPG & 1705 & $33.0 \pm 5.0$ \\
\hline Quantification of the four different classes of retrotransposons discovered as part of this study. Rows in italic indicate totals across the classes listed above
\end{tabular}



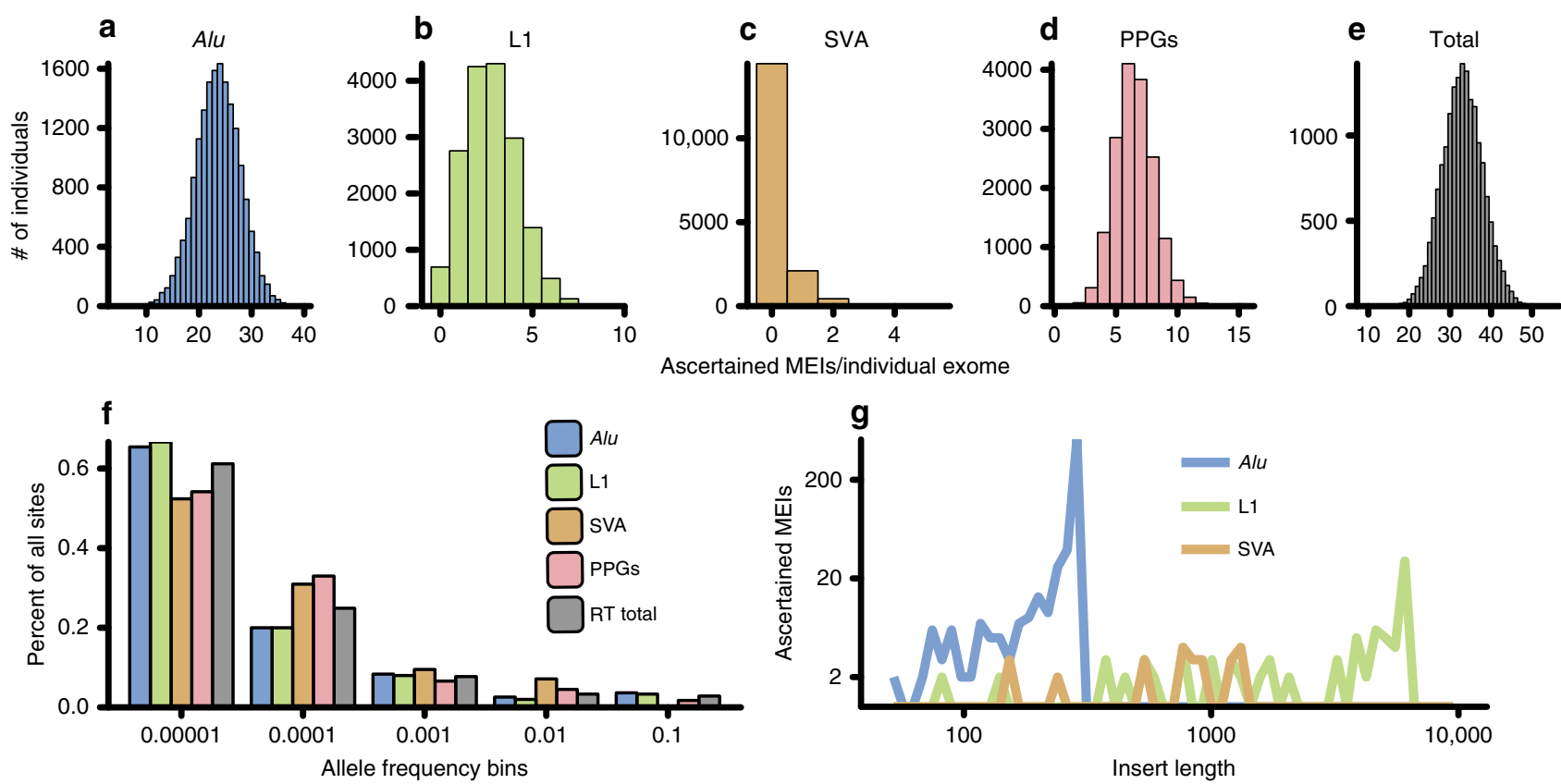

Fig. 1 The DDD RT call set. a-e Histograms of the total number of variants per individual for the four classes of RT events identified in the DDD cohort (Alu: blue; L1: green; SVA: orange; PPGs: red; combined RT events: grey) in size one bins. f Allele frequency distributions for the RT classes depicted in a-e in $\log _{10}$ allele frequency bins. $\mathbf{g}$ Insert size estimates provided by MELT for the MEl classes ascertained in this study in $\log _{10}$ insert size bins. All plots only include variants from unaffected parents

mosaic. Using long-PCR coupled to long-read sequencing (see methods), we were able to phase seven of our false-negative $A l u$ variants to nearby SNVs. With our approach, we determined that one was part of a complex event of unknown mosaicism (FCGBP: c.4743_4744insAlu(1_110)), and six were constitutive in both parent and proband (Supplementary Fig. 2; Supplementary Data 2).

As expected, the total number of variants per individual for each RT class (Fig. 1a-d) as well as combined number of RT events (Fig. 1e) approximated a Poisson distribution (see the Methods section; Supplementary Fig. 3). The vast majority of variants are rare (allele frequency $<1 \times 10^{-4}$; Fig. 1f), with $>65 \%$ of Alu and L1 variants identified in fewer than four unrelated individuals. SVA and PPGs appear to be moderately under ascertained compared with Alu and L1 at lower AFs, with $>50 \%$ of variants identified in the lowest AF bin and length estimates for the three MEI classes largely fit the findings of previous studies (Fig. 1g) ${ }^{4,5}$.

We next sought to ensure that our total number of ascertained RT variants, both on a population and individual basis, accorded with previously published WGS data ${ }^{4,5}$. On a population level, WES did not appreciably limit our overall sensitivity compared with the WGS sampled data. When we compared a downsampled version of our call set to the 1KGP, our total number of Alu and SVA variants fell within the expected distribution, while L1 was close to expectation (Supplementary Fig. 4).

To assess the quality of the PPG call set, we compared PPG allele counts (i.e., total number of individuals with a retroduplication of a given gene) to a recent assessment of PPGs in samples sequenced as part of the $1 \mathrm{KGP}^{6}$. Generally, PPGs identified in both data sets shared similar relative allele counts $\left(r^{2}=0.64\right)$ and variants identified in this study, but missing from Zhang et al. ${ }^{6}$ are typically rare (Supplementary Fig. 5). To further validate our approach and ensure that the identified PPG donor genes fit with previously identified patterns of germline PPG formation ${ }^{2,19}$, we assessed each donor gene for both functional annotation and expression across 30 tissue types analyzed by the GTEx consortium ${ }^{20}$. The major functional cluster $\left(D_{A V I D}{ }^{21}\right.$ enrichment score 8.82 ) belonged to genes involved in the ribosomal and translational machinery, consistent with previous findings involving fixed PPGs in the human genome ${ }^{2}$. Our expression analysis likewise confirmed previous findings ${ }^{19}$, and shows that donor genes that give rise to PPGs are more highly expressed in a large number of tissues compared with non-retroposed genes (Wilcoxon rank sum $p<1 \times$ $10^{-3}$ for all tissues; Supplementary Fig. 6). In addition, while it could be assumed that increased germ-line expression of a gene may play a role in increased probability of PPG generation, when we compared PPG donor gene expression in the testis and ovary to that in other tissues, the majority of tissues (19/28, identical tissues for ovary and testis) showed statistically identical patterns of donor gene expression (Wilcoxon rank sum $p>1 \times 10^{-3}$; Supplementary Fig. 6).

Coding RT burden and constraint. As expected for WES, the vast majority $(84.9 \%)$ of detected MEIs impacted the coding or intronic sequence of a protein-coding gene or a regulatory element targeted in the augmented WES assay described in Short et al. ${ }^{15}$ (Fig. 2a). Due to the large numbers of individuals with WES data in this study, we have ascertained over five-fold more exonic variants than the largest previously published study (Supplementary Fig. 7) ${ }^{4,5}$. Nonetheless, the number of MEIs identified in this study, based on the proportion of the genome assayed, likely represent only $2.2 \%$ of MEI variants genome wide in these individuals.

Our large collection of coding variants allowed us to examine the evolutionary forces acting on coding MEI variation (Fig. 2b). To examine selective constraint, we utilized two common measures: the proportion of variants observed in only one individual (e.g., singletons) ${ }^{22}$ and the proportion of variants found in genes likely to be intolerant of loss of function (LoF), as determined by the pLI score ${ }^{23}$. To avoid issues of relatedness and the potential for clinical ascertainment bias for pathogenic MEIs in individual DD patients, only the 17,032 unaffected parents sequenced as part of DDD were included in our analysis. MEIs which directly impact exons are under strong selective constraint, 
a

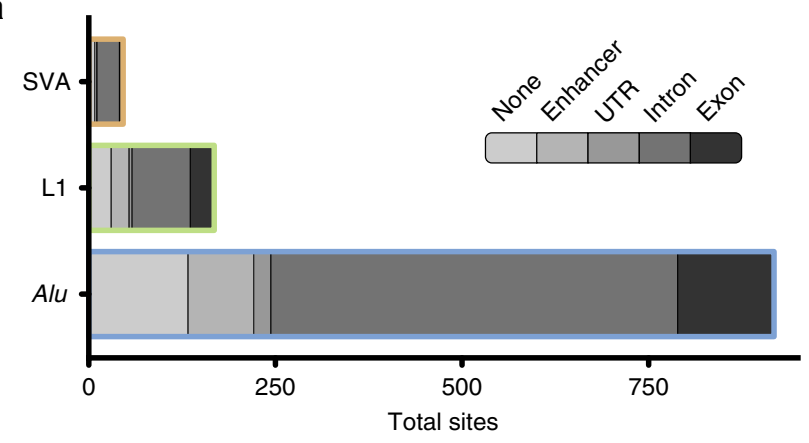

b

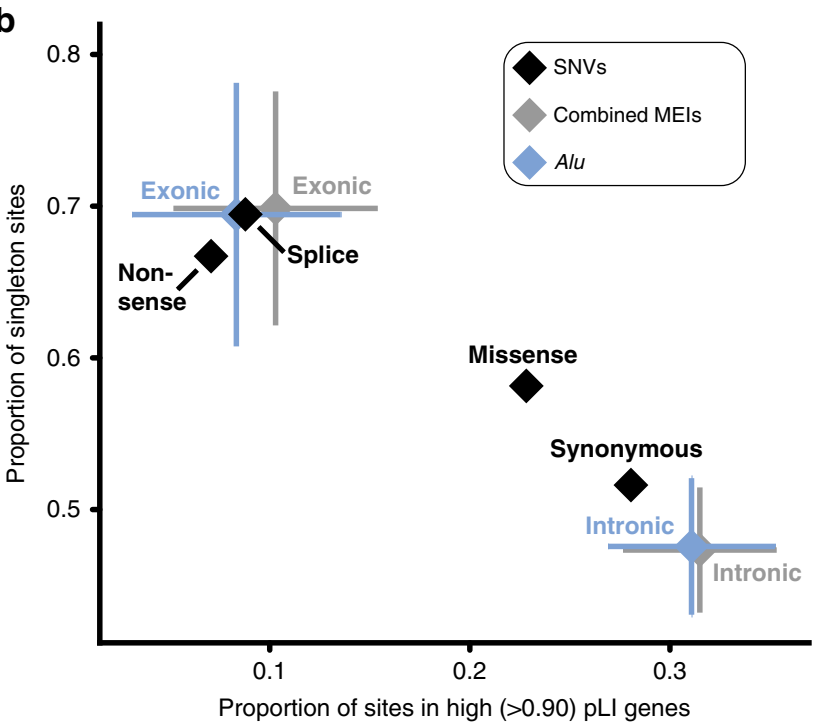

Fig. 2 Coding constraint on MEls. a Cumulative consequence annotations for Alu, L1, and SVA MEls in all samples ( $n=28,132$ individuals) analyzed. The majority of variants identified in this study fell within the noncoding space (either an enhancer or intron). b Comparison of constraint between MEls and SNVs in unaffected parents. To compare the impact of exonic and intronic Alu (blue) and all MEls (grey) to varying classes of SNVs (black), we used two metrics: the proportion of variants in genes that have been identified as LoF intolerant as gauged by $\mathrm{pLI}$-score 22 ( $x$-axis) and the proportion of variants identified in only one individual (i.e., singletons; $y$ axis). Error bars indicate $95 \%$ confidence intervals based on population proportion; confidence intervals were calculated for SNVs, but are too small to appear at the resolution displayed in this figure

indistinguishable from that of both nonsense and essential splice site SNVs (Fig. 2b). Interestingly, we did not find any sign of selection acting on intronic MEIs as they appear to be constrained similarly to synonymous SNVs. In contrast to previous studies 24,25 , we did not find a statistically significant $\left(\chi^{2} p<\right.$ $0.05)$ bias toward intronic MEIs inserted in the antisense orientation of the gene in which they are found (Supplementary Fig. 8). This is likely not a repudiation of such work, but attributable to the relatively small number of intronic events we identified as part of our analysis compared with $\mathrm{WGS}^{4,24}$ or reference genome-based ${ }^{25}$ studies. To put our findings on exonic MEI constraint into perspective with other forms of variation, every human genome will harbor approximately one $(0.76 \pm 0.62$ SD per individual) MEI which directly impacts protein-coding sequence. Since MEIs are similar to nonsense SNVs in terms of deleteriousness (Fig. 2b), MEIs thus make up $0.6 \%^{22,26}$ of all coding protein-truncating variants (PTVs; among SNVs, InDels, and large $\mathrm{CNVs}$ ) in each individual human genome.
While we were unable to perform similar population genetic analyses for PPG events, due to the difficulty of resolving the putative insertion site with WES data and thus distinguishing between different PPGs for the same donor gene, we were able to assess the propensity for specific genes to give rise to PPGs based on their selective constraint. We observed that PPG donor genes were significantly enriched for genes that are highly intolerant of loss of function variation ( $\mathrm{pLI}>0.9$ ). High pLI genes make up $25.3 \%$ of donor genes, compared with $17.6 \%$ of all protein-coding genes (Fisher's $\left.p=2.6 \times 10^{-4}\right)^{22}$. This observation is likely driven by loss-of-function intolerant genes being more likely to be highly expressed in multiple tissues ${ }^{22}$, similar to genes known to have been retroduplicated (Supplementary Fig. 6) ${ }^{19}$. This observation implies that PPG events rarely strongly perturb the function of their donor gene-despite several previously documented instances of PPGs impacting expression or functionality of their donor gene ${ }^{27}$.

Clinical annotation of de novo RT variants in DD. Using the computational approaches outlined above, we identified a total of 11 germ-line de novo RT variants (Table 2). Our findings include coding, noncoding, pathogenic, and benign variants, as well as, to our knowledge, the first de novo MEI identified in a pair of monozygotic twins (Supplementary Fig. 9). All de novo RT variants were confirmed via a PCR assay specific to the RT class (Fig. 3; Supplementary Fig. 9; Supplementary Fig. 12) and, where possible, inspected for poly(A) tail and target site duplicationhallmarks of bona fide RT activity ${ }^{28}$. We identified no de novo RT variants which localized to the noncoding elements included on the WES capture, which falls in line with expectations based on mutation rate estimates (Fig. $4 \mathrm{~b}$ ). We also attempted to determine the parental origin of each RT event using SNVs located on sequencing reads which support the RT insertion (Table 2). Of the 11 de novo RT events, we were able to phase three variants, all to the father. While this finding is not statistically significant $\left(\chi^{2}\right.$ $p=0.083)$, it fits with previous findings that the majority of de novo structural variants ${ }^{9}$, and indeed most variant classes ${ }^{29}$, are attributable to paternal origin.

Nine of our validated de novo mutations were MEIs ( $7 \mathrm{Alu}$ and 2 L1), or a rate of approximately one de novo event per every 1000 patient exomes sequenced (9/9738). As expected, based on both the total number of polymorphisms $\mathrm{s}^{3-5}$ and mutation rate (Table 1; Supplementary Table 3), we identified more Alu de novo variants than the other RT classes. We also identified two PPG germ-line de novo variants, or approximately one new PPG per every 5000 patient WGS (2/9738). As a further quality control for PPGs, we capillary sequenced all resulting PCR products to confirm the gene of origin (Supplementary Fig. 12) and performed WGS to identify the PPG insertion site. We were able to localize the SERINC5 PPG to an $\sim 50 \mathrm{Kbp}$ intron of the gene CLIC4 and the SLC35F2 event to an intergenic region between the genes MAK and GCM2 (Fig. 3e). Neither of the events directly impacted coding sequence and CLIC4 is neither under strong selective constraint nor known to have any link with DD.

Each de novo mutation was then compared with known DDassociated genes (using the Developmental Disorders Genotypeto-Phenotype database-DDG2P) to identify potentially pathogenic variants (Table 2). Of the mutations identified, four directly inserted into coding exons of DD-associated and fetal brainexpressed (see the Methods section; Supplementary Table 4$)^{30}$ genes (Fig. 3, Table 2) with all four found in genes statistically enriched for PTVs ${ }^{12}$ and therefore likely to operate by a LoF mechanism. We did not identify any intronic de novo mutations likely to be pathogenic (Fig. 3a-d; Supplementary Fig. 9). An additional mutation inserted into the coding sequence of a 


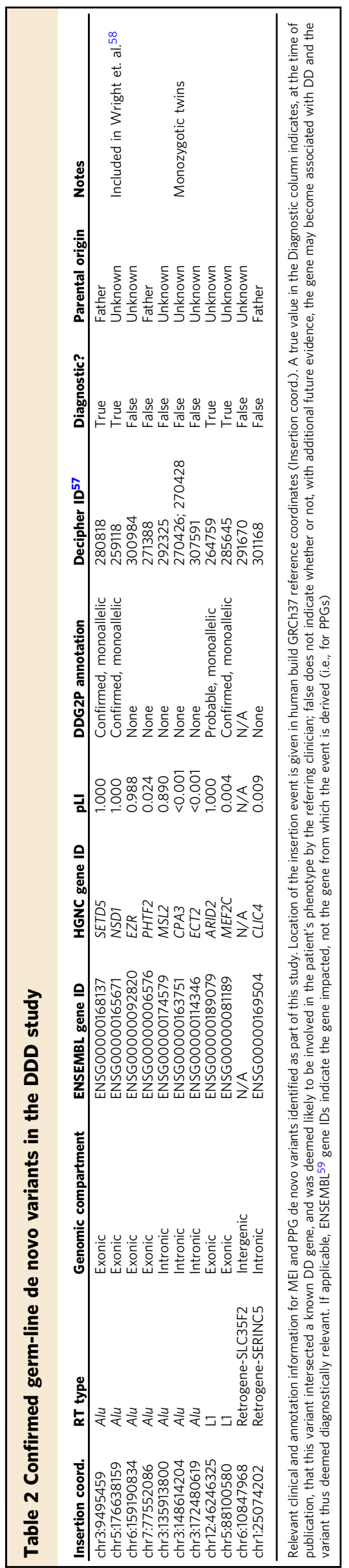

strongly LoF-intolerant gene, EZR ( $\mathrm{pLI}=0.99$; Supplementary Fig. 9), but we could not directly attribute it to the patient's phenotype due to lack of significant enrichment for PTVs, although there is prior evidence for a role in a familial DD syndrome $^{31}$. The four mutations in $\mathrm{DD}$-associated genes were reported to the referring clinician for clinical interpretation based on both initially reported and updated phenotypes (Supplementary Table 2). Three out of four reported mutations (NSD1, $M E F 2 C$, and ARID2) were subsequently deemed to be likely causative of the patient's phenotype (Supplementary Table 2) by the referring clinician. To determine if our patient with a likely pathogenic NSD1 variant phenocopies the already described aberrant blood DNA methylation signature typical of Sotos syndrome patients ${ }^{32}$, we compared our patient's genome-wide methylation status to several already published Sotos syndrome cases (see the Methods section) ${ }^{32}$. Our patient strongly clusters with other confirmed Sotos syndrome patients and confirms our clinical assessment and diagnosis of this particular patient (Supplementary Fig. 10). The fourth patient, with an Alu insertion in SETD5 (Fig. 3a), has clinical features (polydactyly and truncal obesity; Supplementary Table 2) more suggestive of a ciliopathy. As such, the identified MEI is unlikely to be the sole cause for the patient's DD, but may contribute to a composite phenotype. Accordingly, we did investigate this patient's exome for other potentially diagnostic variants, but did not identify additional de novo or inherited variation that could plausibly be associated with this patient's phenotype.

We also examined our data set for inherited rare pathogenic RT variants. We evaluated variants inherited from an affected parent, bi-allelic inheritance (either a homozygous MEI or a heterozygous MEI paired with another variant class), and Xlinked variants maternally inherited by affected males. We did neither identify any rare MEI variants inherited from an affected parent nor any compound heterozygous individuals with a rare MEI and a non-MEI PTV (e.g., SNV/InDel) impacting the same gene. We did identify a single proband-specific homozygous MEI inserted into an exon of PAN2 which was unique to a single family. This gene was recently identified as nominally significant (genome wide $p=4.2 \times 10^{-4}$ ) in a study investigating the role of recessive variants in $\mathrm{DD}^{13}$, although more data are required to be confident of its association to DD. We also identified a total of 22 (14 Alu, $7 \mathrm{~L} 1$, and 1 SVA) polymorphic MEIs on the $\mathrm{X}$ chromosome, of which 4 (3 Alu and $1 \mathrm{~L} 1)$ directly impacted protein-coding sequence. Of these variants, none were at a low enough allele frequency to be reasonably DD associated, were located within a gene associated with $\mathrm{DD}$, nor fit an inheritance pattern consistent with $\mathrm{X}$-linked disease.

Mutation rate and enrichment of deleterious $\mathrm{RT}$ events in DDD. Based on our findings, in the coding and peri-coding portion of the genome, one out of every $2434 \mathrm{DD}$ cases $(0.04 \% \pm$ $0.04 ; 95 \% \mathrm{CI})$ is directly attributable to RT-derived mutagenesis. To determine both if our observed number of de novo variants meets expectation and if our patient cohort is enriched for causal de novo RT events, we estimated the population mutation parameter, $\Theta^{33}$, from the unaffected parents in the DDD study and from the $1 \mathrm{KGP}^{4,5}$ (Supplementary Table 3 ). The resulting calculation gives very similar estimates of MEI mutation rate (combined across Alu, L1, SVA) of between $1.4 \times 10^{-11}$ (1KGP) and $1.2 \times 10^{-11}$ (DDD) variants per bp per generation $(\mu)$, or $\sim 1$ new MEI genome-wide per every $12-14$ births-largely concordant with prior estimates from WGS data sets $3,34,35$.

Using this genome-wide mutation rate, we estimated the number of expected mutations in various genomic compartments, including within genes intolerant to PTVs and within 


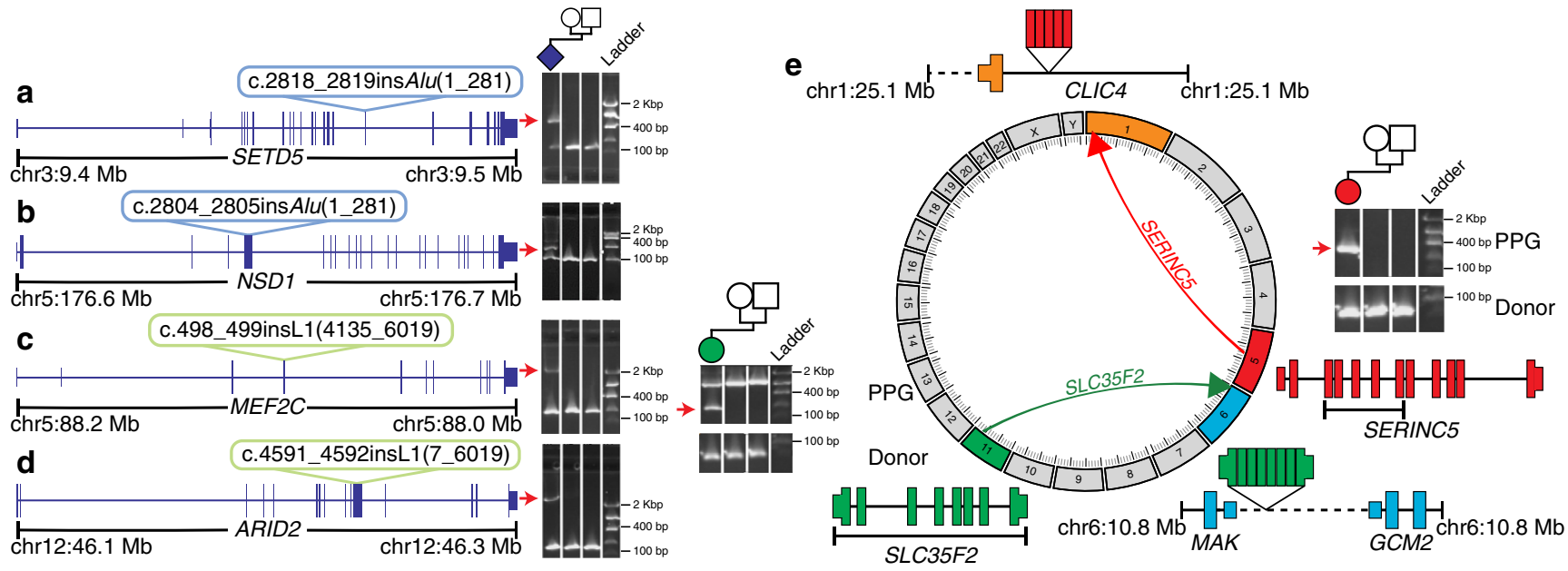

Fig. 3 RT-derived de novos in the DDD. We identified a total of nine de novo MEls, four of which disrupted the protein-coding sequence of a known DD gene: a SETD5, b NSD1, c MEF2C, and d ARID2. Shown in each panel is a diagram of the affected gene (blue model) with the relevant insertion indicated with a colored bubble. To the right are PCR validations confirming the de novo status of each mutation; a positive result is indicated by a raised secondary band present only in the proband sample (red arrow). e Circos diagram and PCR results for two identified germ-line de novo PPGs. For each de novo PPG shown is a diagram of the donor gene (gene model), location of duplication as PPG (directional arrow), and new insertion site. Exons from the donor gene included in the PPG are indicated by brackets underneath the donor gene model. To confirm PPG presence, PCR was performed (Methods) on proband, paternal, and maternal gDNA (sample in each lane is shown by pedigree). The band which represents the PPG is marked with a red arrow, and was confirmed via capillary sequencing (Supplementary Fig. 12). Dashed lines indicate intergenic regions, all genes models are shown in sense orientation, and PPG gene diagrams are not to scale

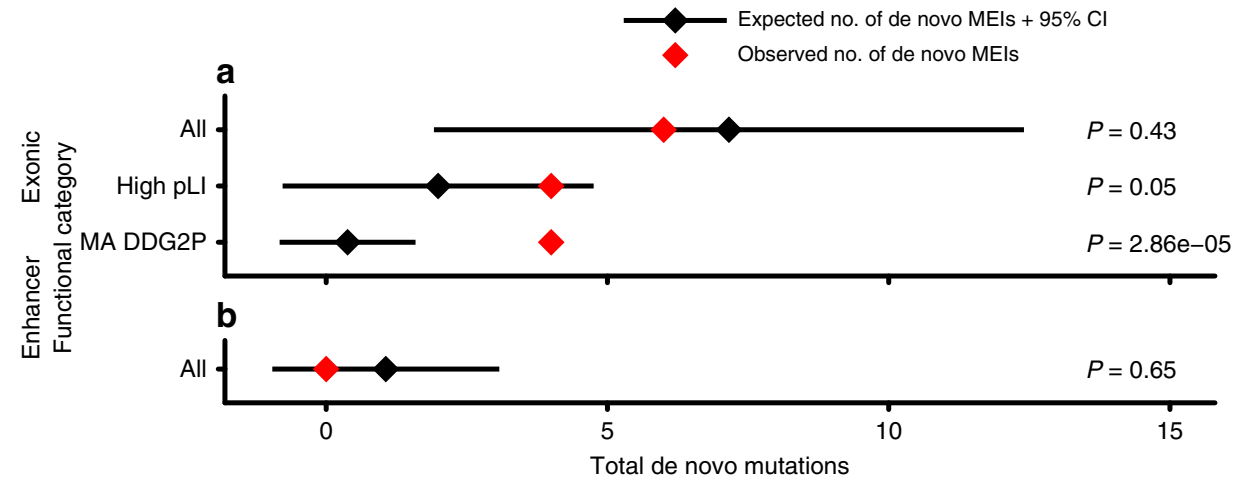

Fig. 4 Estimating enrichment of deleterious MEls. Depicted are the total number of expected (black) and observed (red) de novo mutations observed in exons (a) and enhancers (b) for all, high pLI ( $\mathrm{pLI}>0.9$ ), and known monoallelic DD (MA DDG2P) genes. Expectation is based on the Poisson distribution of 100 simulations utilizing the neutral mutation rate $\left(1.2 \times 10^{-11} \mu\right)$. P-values are based on the Poisson distribution and used to determine statistical deviation of observed to expected de novo counts for exons and enhancers

DD-associated genes (Fig. 4; Methods). We identified a significant enrichment of de novo MEIs in dominant DD-associated genes (Poisson $p=2.86 \times 10^{-5}$ ), but not in the much larger set of LoF intolerant genes (Poisson $p=0.05$ ). To ensure that this finding was not due to inaccurate estimation of the genome-wide mutation rate, we also assessed the probability that four out of six exonic de novo MEIs would fall within exons of dominant $\mathrm{DD}$-associated genes by chance, based on the proportion of the exome represented by these genes (and assuming known DDassociated genes have the same MEI mutation rate as other genes) and likewise found a significant enrichment $\left(p=4.3 \times 10^{-5}\right.$; see the Methods section).

\section{Discussion}

Here, we have described the development, validation, and exemplification at scale of an analytical pipeline for the rapid assessment of patient genomes for RT variants. We have used these approaches to present the largest study examining the coding genome for RT-derived variation to date (Table 1; Fig. 1). With this data set, we first demonstrated that exonic MEIs (regardless of insertion length) are under selective constraint on par with protein-truncating SNVs (Fig. 2). We identified four likely pathogenic RT mutations, two Alu and two L1 insertions (Fig. 3), all of which arose de novo in known haploinsufficient DD-associated genes (Fig. 3a-d), implying that dominant loss-offunction is the major mode of pathogenic exonic RT variation. Finally, we estimated the genome-wide MEI mutation rate and used it to determine that DDD probands are enriched for damaging RT variation within exons of dominant DD-associated genes (Fig. 4a).

The total number of polymorphic, exonic RT variants identified in DDD is concordant with previous studies characterizing MEI variation ${ }^{3,5,36}$. Pathogenic MEIs make up $0.04 \%$ of diagnoses in the DDD study (4/9,738 probands), a small yet individually significant collection of diagnostic variants. We additionally investigated our data for causative, inherited MEIs, and did not identify any such events. We infer that despite making up a 
significant proportion of reported MEI variants in the clinical literature ${ }^{7}$, bi-allelic or X-linked MEI events are a less frequent class of pathogenic variant in developmental disorders. This is in keeping with recent estimates ${ }^{13}$ that in a largely outbred clinical population, such as in the UK, recessive disorders caused by coding variants account for a much smaller fraction of patients than dominant disorders. We also attempted to investigate both the parental origin of de novo variants (Table 2) and the mosaicism of MEIs with low variant allele frequency (Supplementary Fig. 2). In both cases, additional work incorporating long-read technology is needed to ascertain parental contribution and, in the case of parental mosaic MEIs, potential recurrence risk.

Interestingly, it appears that the contribution of diagnostic RT variants may vary among diseases. Wimmer et al. ${ }^{37}$ reported a total of 13 diagnostic, exonic MEI variants in 4500 neurofibromatosis type I patients ( $0.3 \%$ of patients). This rate is seven times higher than that observed in DDD, and was attributed to a potential RT mutation hotspot associated with the canonical L1 endonuclease cleavage site of $3^{\prime}$-AA/TTTT- $5^{\prime 38}$ within the neurofibromatosis-associated gene, NF1. A recent study using a cell culture retrotransposition assay to study MEI site preference was unable to identify a mutation hotspot in $N F 1^{39}$, and therefore suggests further work is needed to investigate the role of sequence context in determining the overall genomic landscape of RTmediated disease. Analogously, inclusion of sequence context into the SNV mutation model noticeably improved the ability to determine enrichment/depletion of deleterious SNVs within genes $^{22,23}$.

Our study is limited in that by analyzing exome sequences we only identified $\sim 2 \%$ of the RT variants in each individual human genome studied ${ }^{4,5}$. Despite a number of known disease-associated intronic MEIs in the literature, we did not identify a pathogenic intronic MEI. As such, it remains an open question as to what contribution RT mutations in the noncoding genome play in the etiology of DD. While it appears that the contribution of regulatory elements to DD is relatively small, as defined by this (Fig. 4b) and other studies ${ }^{15}$, previous work has identified a significant signature of purifying selection against MEI events within $100 \mathrm{bps}$ of exons ${ }^{25}$-variants which our study could potentially identify. As our data suggest that the majority of DD cases with pathogenic coding MEIs are due to de novo insertions (Table 2; Fig. 3), we conjecture that most additional DDassociated MEIs may be located in the introns of known DDcausing genes and disrupt splicing-a known disease mechanism attributable to RT-derived mutagenesis ${ }^{7,37,40}$. Simulations suggest that under a null genome-wide mutation model, we should expect to observe 12.3 (5.4-19.2, 95\% CI) de novo intronic RT mutations in dominant DD-associated genes in a population sample of 9738 individuals. As such, a WGS study of a clinical population of similar size to that analyzed here should be well powered to estimate the pathogenic contribution of intronic MEIs.

De novo MEIs are typically readily interpretable with modest informatics expertise, and represent a clinically relevant class of variation to assay in clinical bioinformatics pipelines. While we ultimately find that the overall burden of RT-attributable disease is relatively low in the human population, it is nonetheless an important consideration when elucidating the genetic basis of disease in individual patients.

\section{Methods}

Patient recruitment and sequencing. A total of 13,462 patients were recruited from 24 clinical genetics centers from throughout the UK and the Republic of Ireland as previously described ${ }^{41}$. Informed consent was obtained for all families, and the study was approved by the UK Research Ethics Committee (see the Acknowledgements section). For the purposes of this study, individuals that were not recruited as part of a trio (e.g., individual patients or patients with just one parent), were included on the DDD sample blacklist, or failed to meet MELT QC requirements ${ }^{5}$ were excluded from downstream analysis (leaving $n=9738$ probands; 28,132 individuals). Sequencing of families and alignment to build GRCh37 of the human reference genome with bwa ${ }^{42}$ were performed as previously described ${ }^{12}$.

Processed pseudogene pipeline development. PPGs, particularly young polymorphic events, share highly homologous sequence with the source gene from which they are derived. Consequently, the WES bait capture method will capture both DNA from the original donor gene and the new daughter copy. This allows, compared with our approach for MEI discovery, for ascertainment of PPGs genome wide. While this approach does come with limitations, such as difficulty in identifying insertion variants, we can still determine events per individual.

Our discovery pipeline functions in two steps: first we collect read evidence on an individual level to determine which genes have been retroduplicated in that individual (Supplementary Fig. 1). Second, we determine presence/absence of each PPG in every individual in the DDD cohort based on the gene models built in the first step. In step one, we iterate over all genes in the ENSEMBL gene database which have a determined pLI score ${ }^{22}$ and collect discordant read pairs (DRPs) which map between exons and have an insert size $>99.5 \%$ of all other reads in the sample. If more than four reads linking two exons are found, the gene is considered to be retroduplicated elsewhere in the genome. In step two, for each gene identified in step one, all evidence across all PPG-positive individuals are pooled to make a model of the PPG. This model is then used to check for DRP and split read pair (SRP) evidence in all genomes. If an individual has at least five total read pairs of supporting evidence with at least one SRP and one DRP, an individual is considered positive for the given PPG. All genes and individuals were combined into a flat file listing presence or absence of a given PPG in each individual.

MEI call set generation and consequence annotation. To identify MEIs in the DDD WES data, we utilized the previously published MELT ${ }^{5}$. MELT was run with default parameters (except the "-exome" flag during IndivAnalysis) using "Split" mode to generate a final unified VCF-format file ${ }^{43}$ of all 28,132 unfiltered individuals independently for each MEI type $(A l u, L 1, S V A)$. Following initial data set generation, we found that a subset of variants internal or adjacent to $( \pm 50 \mathrm{bp})$ low complexity repeats (defined here as a run of sequence $\geq 15$ bp composed of two or fewer nucleotides) were likely false positive. As such, we added an additional filter to the final MELT VCF, lc (low complexity), which removes such false positives from downstream analysis. Variants that could not be genotyped in at least $25 \%$ of individuals, had $\leq 2$ split reads, had MELT ASSESS score $<3$, or had any value in the VCF FILTER column other than PASS or rSD were filtered.

To determine if variants per individual approximated a Poisson distribution, we calculated the mean number of sites per unrelated individual $(\lambda)$ independently for each RT class and for all RT classes combined as displayed in Fig. 1a-e. Our calculated $\lambda$ was then supplied to the function rpois in $\mathrm{R}$ to generate a random deviate and plotted alongside histograms identical to Fig. 1a-e in Supplementary Fig. 3.

To generate consequences plotted in Fig. 2a, all MEIs were annotated using Variant Effect Predictor v88 (VEP) ${ }^{44}$ on human genome build GRCh37 and intersected with bedtools intersect ${ }^{45}$ to enhancers (one of heart ${ }^{46}$, VISTA ${ }^{47}$, or highly evolutionarily conserved ${ }^{48}$ ) included on the DDD WES capture ${ }^{15}$. Only a single consequence was retained for each variant, with priority given to enhancer annotation. Primary transcript as determined by VEP was used for all gene-based consequences, pLI score ${ }^{22}$ annotation, and DDG2P disease association (Table 2).

Assessment of potentially mosaic MEls. To determine the mosaicism of variants that were identified as false-negative de novo during initial MELT analysis (i.e., genotyped as heterozygous in a single proband and homozygous reference in both parents), we randomly selected 1000 trios and determined the total number of reads supporting both the insertion and reference allele (i.e., allelic proportion) at all $917 \mathrm{Alu}$ loci identified in this study. Alongside this data, we plotted the parental allelic proportion at the 15 false-negative Alu loci described above (Supplementary Fig. 2a). To formalize this result and ensure that these specific loci do not have higher genotyping or sequencing error than other loci, we performed three analyses. First, we compared the combined allelic proportion of all heterozygous variants to the combined allelic proportion of both false-negative parents and the associated heterozygous probands (Supplementary Fig. 2b). Second, we examined the allelic proportion for all homozygous reference genotypes at each false-negative locus for evidence of higher error rates based on higher average allelic proportion at those loci using a z-test (Supplementary Fig. 2c). Finally, to assess if these loci had similar read start distributions in both parent and proband, we retrieved all read start sites within 250 bps of each insert site and used a Kolmogorov-Smirnov test to compare the two distributions. Only one locus, chr11:g.95523918_95523919insAlu (1_280), showed a significant difference at a threshold of $p=0.05$ (K.S. test $p=$ $\left.2.70 \times 10^{-4}\right)$.

Since these sites are not likely to be genotyping or sequencing artifact, we next attempted to clarify the nature of a subset of variants with a custom long-range PCR and Pacific Biosciences sequencing approach. The goal of this methodology 
was to attempt to both validate and phase candidate mosaic MEIs to nearby $( \pm 5$ Kbps) SNVs. As above, we limited our analysis to Alu MEIs as the large insert length of L1 $(n=1)$ and repeat heavy SVA $(n=2)$ elements would likely prove refractory to long PCR with enough flanking sequence to phase to nearby SNVs. To design locus specific primers, the genomic region surrounding each target MEI was entered into Primer-BLAST [www.ncbi.nlm.nih.gov/tools/primer-blast/] using default parameters. Each locus was targeted with three primer pairs: one pair amplifying a $10 \mathrm{~kb}$ region with the MEI lying centrally, and two pairs of primers to amplify $5 \mathrm{~kb}$ fragments extending up and downstream of the MEI. In total, $10 \mathrm{~kb}$ PCRs were performed using AccuTaq LA DNA Polymerase (D8045, SigmaAldrich) with the following parameters: $98^{\circ}$ denaturation for $1 \mathrm{~min}$, followed by 30 cycles of $94^{\circ}(30 \mathrm{~s}) / 54^{\circ}(30 \mathrm{~s}) / 68$ degrees $(11 \mathrm{~min})$, with a final extension of $18 \mathrm{~min}$ at $68^{\circ}$. Total $5 \mathrm{~kb}$ PCRs were performed as above, but with a reduced $6 \mathrm{~min}$ extension time at $68^{\circ} \mathrm{s}$, and a final extension time of $10 \mathrm{~min}$. After quality checking the PCRs on control DNA, 100-200 ng of both parental and proband DNA were amplified in a $50 \mu \mathrm{l}$ PCR reaction using the same parameters.

After confirming amplification by agarose gel, PCR products were isolated using SPRI bead purification (Ampure-XP, Agilent), quantified, and mixed in equimolar amounts. We then performed a standard PacBio library prep using SMRTbell Template Prep Kit 1.0 (part no. 100-991-900) without size selection. Sequencing was performed on the Pacific Biosciences Sequel platform using Sequel Sequencing Kit 3.0 (101-597-800) and Sequel SMRT cell $1 \mathrm{M}$ v3 (101-531-000). For error correction, raw sequencing reads were then provided as input to pbccs v3.4.1 [https://github.com/PacificBiosciences/unanimity] with default parameters other than minLength $=100$. Corrected reads were then aligned to the human genome using blasr v5.3.3 $3^{49}$ and downsampled to between $2000-3000 \times$ coverage per locus assessed.

MEIs were then phased to the nearest heterozygous SNV on a per-read basis using a custom java pipeline. After dropping sites due to PCR errors or phasing ambiguity, we were able to examine a total of $6 \mathrm{Alu}$ MEIs (Supplementary Data 2). The total number of reads supporting each of four possible phases (MEI + SNV +, MEI + SNV-, MEI-SNV +, MEI-SNV-) were then quantified. Primers and quantification of the results are available in Supplementary Data 2.

Quality control of RT data using WGS and 1KGP. To determine if our MEI WES call set was biased compared with WGS data, we performed two independent comparisons: (1) to high coverage $(>30 \times)$ WGS data generated for a subset of DDD trios; and (2) to a published collection of MEIs from 1 KGP phase $\mathrm{III}^{5}$.

For WGS quality control, we used a subset of $30 \mathrm{DDD}$ trios $(n=90$ individuals), which were previously whole-genome sequenced. MEI discovery using MELT $^{5}$ on all 90 individuals was performed and filtered identically to the WES data. Genotypes identified in the WGS data but not in WES were then separated based on coverage in the corresponding WES. Genotypes in low coverage areas (< $10 \times$ ) were considered not possible (n.p), while variants where coverage was greater than 10x are considered not detected (n.d). All remaining genotypes were than compared for identity between the WGS and WES results (Supplementary Table 1).

To compare the DDD MEI call set to the 1KGP, we first filtered 1KGP calls to variants with mean $>10 \times$ coverage in 1000 randomly sampled DDD WES individuals (leaving $318 \mathrm{Alu}, 81 \mathrm{Ll}$, and $26 \mathrm{SVA}$ ). We then randomly selected 2453 DDD parents 1000 times, retaining only loci present in downsampled individuals ${ }^{4,5}$. The resulting distribution was then compared with the observed number of variants in the 1 KGP-masked data to generate z-scores independently for all three MEI types (Supplementary Fig. 4).

To compare our PPG data set to Zhang et al. ${ }^{6}$, we downloaded provided Supplementary tables. We then summed the total number of unique events per person, and determined counts for each gene reported. Genes were then matched between our call set and Zhang et al. ${ }^{6}$ using ENSEMBL gene identifiers, and allele counts between each data set were plotted to create Supplementary Fig. 5.

GTEx annotation of processed pseudogenes. To determine RNA expression levels of donor genes which gave rise to PPGs identified in this study, we queried transcript per kilobase per megabase of sequencing (TPM) scores for all genes in 30 tissues assessed by the current GTEx v7 release [https://gtexportal.org/home/ datasets]. Only the 18,225 protein-coding genes which were assessed for gene PPGs by our project were retained for subsequent analysis. TPM values were then averaged across all GTEx individuals for a given tissue to generate a mean TPM value as plotted in Supplementary Fig. 6. Nonparametric Wilcoxon rank-sum tests were performed using the wilcox.test function in $\mathrm{R}$ with default parameters to generate $p$-values for both within tissue and between tissue comparisons.

SNV calling and quality control. To call SNVs from all DDD individuals, we utilized GATK 3.5-0-ge91472 $\mathrm{d}^{50}$ in three steps using default settings. First, we called variants in individual samples using HaplotypeCaller. Next, individual VCF files were processed in 200 individual batches using CombineGVCFs. Finally, all batched VCFs were passed to GenotypeGVCF to generate a final joint-called VCF file. This file was then annotated using VEP v8 $8{ }^{44}$ on build GRCh 37 of the human genome; final annotations were determined only for the canonical transcript as determined by VEP. Unaffected parents ( $n=17,032$ individuals) were then extracted from this VCF and only variants with an allele count $\geq 1$ in these individuals were retained.

For initial filtering, we removed SNVs with a VQSLOD $<-2.0$, read depth $<8$, and genotype quality $<20$. We next performed more extensive QC using a "missingness" score identical to the method described in Martin et al. ${ }^{13}$. In short, each genotype at a given variant was assessed for genotype quality (GQ), depth (DP), and a binomial test for allelic depth (i.e., number of alternate vs. reference supporting reads; $\mathrm{AD}$ ). If a given genotype had $\mathrm{GQ}<20, \mathrm{DP}<8$, or $\mathrm{AD} p$-value $<$ 0.001 , it was considered "missing". If more than $50 \%$ of genotypes for a given variant were missing, the variant was subsequently filtered from final analysis. Allele frequencies were recalculated based on included individuals while accounting for missing genotypes.

Selective constraint of SNVs and MEls. As sensitivity of variant discovery can bias our results, we generated an accessibility mask of the DDD WES data where we expect our variant ascertainment sensitivity to be $>95 \%$ (Supplementary Fig. 11) $)^{5}$. Our mask thus includes only regions of the genome that contain at least $10 \times$ average coverage in a mean cohort of 1000 randomly selected individuals for a total of $74.2 \mathrm{Mbp}$, or $\sim 2.3 \%$ of the genome (Supplementary Table 3). Using this mask, we filtered our original 1129 variants down to $790(653 \mathrm{Alu}, 107 \mathrm{~L} 1$, and 30 SVA) variants in unaffected parents ( $n=17,032$ individuals). Parents were determined to be affected either by the referring clinician or, where ambiguous, through manual curation of HPO terms for a matching parent-offspring phenotype.

Using this masked subset of variants, we determined genomic constraint as shown in Fig. 2b. Allele frequency values were recalculated for all variants, and a pLI score ${ }^{22}$ for each MEI was added as described above. MEIs which did not insert into a gene or inserted into a gene without a calculated pLI score ${ }^{22}$ were excluded from subsequent analysis. We then calculated proportion of singleton variants and proportion of variants in high pLI genes independently for $A l u$ and, due to low overall numbers of the other MEI subtypes, for a combined set of Alu, L1, and SVA. SNVs annotated as nonsense, missense, synonymous, or splice acceptor/ donor (splice in Fig. $2 \mathrm{~b}$ ) as determined by VEP v8 ${ }^{44}$ were extracted from the SNV VCF files described above and used to calculate singleton and pLI proportion identically to MEIs.

Fetal brain expression data. To assess if genes disrupted by RT variants were expressed in the fetal brain, we downloaded per-gene expression data generated by the BrainSpan consortium (file: genes_matrix_csv.zip [http://www.brainspan.org/ static/download.html] $)^{30}$. From this file, we extracted columns representing data generated from fetuses 15 to 37 weeks post conception. These data were then provided as input to a custom python script which calculated the median RPKM of all genes provided for the file. Genes with a median RPKM $\geq 1$ were considered expressed in the fetal brain $(n=15,474)$. Likely causal RT variants $(n=4)$ were then intersected with this list to determine gene expression status. As RPKM $\geq 1$ is a rather liberal cutoff, we also ranked these genes compared with other genes in our expression list; these values are reported in Supplementary Table 4.

MEI validation by PCR. To validate all nine de novo MEI variants (Table 2) and the homozygous insertion in PAN2, we used the following PCR protocol: primers were designed using Primer $3^{51}$ to make products spanning the predicted insertion site (Supplementary Data 1). PCR was carried out using Platinum ${ }^{\text {Tx }}$ Taq DNA Polymerase High Fidelity (Invitrogen); 20 ng of genomic DNA extracted from blood or saliva was amplified in the presence of $0.2 \mu \mathrm{M}$ of each primer and 1 unit of Platinum $^{\text {ma }}$ Taq. Amplification was carried out using the following cycling conditions; for Alu insertions: $2 \mathrm{~min}$ at $94^{\circ} \mathrm{C}$, followed by 36 cycles of $\left(30 \mathrm{~s}\right.$ at $94{ }^{\circ} \mathrm{C}, 30 \mathrm{~s}$ at $60^{\circ} \mathrm{C}$, and $1 \mathrm{~min}$ at $68^{\circ} \mathrm{C}$ ); for LINE1 insertions: $2 \mathrm{~min}$ at $94^{\circ} \mathrm{C}$, followed by 36 cycles of $\left(30 \mathrm{~s}\right.$ at $94^{\circ} \mathrm{C}, 30 \mathrm{~s}$ at $60^{\circ} \mathrm{C}$, and $7 \mathrm{~min}$ at $\left.68^{\circ} \mathrm{C}\right)$. PCR products were visualized using a $2 \%$ agarose $\mathrm{E}-\mathrm{Gel}^{\circledR}$ (Invitrogen).

Processed pseudogene validation. To validate the two de novo PPG variants (Table 2), we used the following PCR protocol: primers were designed using Primer $3^{51}$ to make products within the exons of each gene. Forward and reverse primers were then paired between exons to amplify across the excised intronic regions (Supplementary Data 1). PCR was carried out using either Platinum ${ }^{\mathrm{nx}}$ Taq DNA Polymerase High Fidelity (Invitrogen) or Thermo-Start Taq DNA Polymerase (Thermo Scientific). Platinum ${ }^{\text {mi }}$ Taq assay: $20 \mathrm{ng}$ of genomic DNA extracted from blood or saliva was amplified in the presence of $0.2 \mu \mathrm{M}$ of each primer, and 1 unit of Platinum ${ }^{\text {ms }}$ Taq. Amplification was carried out using the following cycling conditions; $2 \mathrm{~min}$ at $94^{\circ} \mathrm{C}$, followed by 36 cycles of $\left(30 \mathrm{~s}\right.$ at $94^{\circ} \mathrm{C}, 30 \mathrm{~s}$ at $60^{\circ} \mathrm{C}$, and $1 \mathrm{~min}$ at $68^{\circ} \mathrm{C}$ ). Thermo-Start Taq DNA Polymerase assay: $40 \mathrm{ng}$ genomic DNA was amplified in the presence of $0.2 \mu \mathrm{M}$ of each primer and 0.42 units of ThermoStart Taq. Cycling conditions were as follows: $5 \mathrm{~min}$ at $95^{\circ} \mathrm{C}$, six cycles of $(30 \mathrm{~s}$ at $95^{\circ} \mathrm{C}, 30 \mathrm{~s}$ at $64^{\circ} \mathrm{C}$, and $1 \mathrm{~min}$ at $\left.72^{\circ} \mathrm{C}\right)$, six cycles of $\left(30 \mathrm{~s}\right.$ at $95^{\circ} \mathrm{C}, 30 \mathrm{~s}$ at $62^{\circ} \mathrm{C}$, and $1 \mathrm{~min}$ at $\left.72^{\circ} \mathrm{C}\right)$, six cycles of $\left(30 \mathrm{~s}\right.$ at $95^{\circ} \mathrm{C}, 30 \mathrm{~s}$ at $60^{\circ} \mathrm{C}$, and $1 \mathrm{~min}$ at $\left.72^{\circ} \mathrm{C}\right)$ followed by 36 cycles of $\left(30 \mathrm{~s}\right.$ at $95^{\circ} \mathrm{C}, 30 \mathrm{~s}$ at $58^{\circ} \mathrm{C}$, and $1 \mathrm{~min}$ at $\left.72^{\circ} \mathrm{C}\right)$ with a final elongation of $10 \mathrm{~min}$ at $72^{\circ} \mathrm{C}$. PCR products were visualized using a $2 \%$ agarose $\mathrm{E}$ $\mathrm{Gel}^{\odot}$ (Invitrogen). PCR products were sequenced using either the forward or reverse primer used in the amplification protocol by Eurofins GATC Biotech 
GmbH. Sequence traces were aligned using SeqMan Pro 15 (Lasergene 15) and reads were aligned to the human genome (build GRCh37) using BLAT ${ }^{52}$.

WGS of probands with de novo processed pseudogenes. To validate and determine the insertion site of the two identified de novo PPGs (Table 2), we performed Illumina WGS on all individuals of each trio in which the de novo event was identified ( $n=6$ individuals). Samples were first quantified with Biotium Accuclear Ultra high sensitivity dsDNA Quantitative kit using Mosquito LV liquid platform, Bravo WS and BMG FLUOstar Omega plate reader and cherrypicked to $500 \mathrm{ng} / 120 \mu \mathrm{l}$ using Tecan liquid handling platform. Cherrypicked plates are then sheared to $450 \mathrm{bp}$ using a Covaris LE220 instrument and subsequently purified using SPRI Select beads on Agilent Bravo WS. Library construction (ER, A-tailing and ligation) was performed using "NEB Ultra II custom kit" on an Agilent Bravo WS automation system. Samples were then tagged using NextFLEX Unique Dual Indexed adapter 1-96 barcodes at the ligation stage. Libraries were then quantified by qPCR using Kapa Illumina ABI Sanger custom qPCR kits using a Mosquito LV liquid handling platform, Bravo WS, and Roche Lightcycler. Libraries are then pooled in equimolar amounts on a Beckman BioMek NX-8 liquid handling platform and normalized to $2.4 \mathrm{nM}$ for cluster generation on a c-BOT and then sequenced on the Illumina TenX sequencing platform. Following sequencing, reads were aligned with BWA mem $^{42}$ (with settings -t $16-\mathrm{p}-\mathrm{Y}-\mathrm{K} 100000000$ ) to build GRCh37 of the human reference genome. Reads were then manually inspected using the Integrative Genomics Viewer (IGV) ${ }^{53}$ to confirm presence, de novo status, and parent of origin of each PPG.

Methylation analysis. Methylation analyses were performed in accordance with the methods detailed in Choufani et al. ${ }^{32}$. In short, we downloaded publicly available methylation data from GEO (accession GSE74432) generated by Choufani et al. ${ }^{32}$ and combined it with Illumina EPIC methylation array data generated inhouse for our Alu NSD1 loss-of-function patient (Fig. 3b; Table 2). Following data curation, we randomly sampled a subset of 15 cases (defined by Choufani et al. ${ }^{32}$ as a carrier of an NSD1 loss of function variant) and 15 controls to identify a set of differentially methylated signature CpGs based on a $20 \%$ effect size cutoff and 0.01 FDR-corrected $p$-value which separated case and control individuals. Once a signature was established, we used Pearson correlation to assess each sample's similarity to a mean case and mean control based on differential methylation at signature CpG sites. Confirmed pathogenic NSD1 variants and control samples used to build the signature were not included in the correlation analysis (Supplementary Fig. 10).

MEI mutation rate and burden. To determine the mutation rate independently for each MEI type (Alu, L1, SVA), we utilized data generated by both DDD and the $1 \mathrm{KGP}^{5}$. For DDD data, we filtered sites as above based on our $>10 \times$ coverage accessibility mask. For the $1 \mathrm{KGP}$ data ${ }^{5}$, we created a combined mask from three different data sources: (1) the pilot accessibility mask generated by the 1KGP project phase $\mathrm{III}^{54}$, which removes regions of the genome inaccessible to variant calling, (2) reference ME sequences as identified by repeatmasker ${ }^{55}$, as MELT is unable to accurately ascertain MEIs in these regions, and (3) All sequence $\pm 10 \mathrm{Kbp}$ from the $5^{\prime}$ and $3^{\prime}$ terminus of all protein-coding genes from RefSeq ${ }^{56}$. This mask was generated separately for Alu and L1 and did not filter 1113.0 Mbp or 959.9 Mbp of the genome, respectively. The Alu mask was used for filtering SVA and both masks excluded both allosomes. On masking the 1KGP data, we were left with a total of 10,930 autosomal MEIs (8,554 Alu, 2,047 L1, 329 SVA). Following filtering of the DDD and 1KGP sets with their corresponding masks, we used the Watterson estimator with an effective population size of 10,000 for all calculations to estimate the population mutation parameter, $\Theta^{33}$, and mutation rate, $\mu$ (Supplementary Table 3 ).

We next used our estimate of $\mu$ to determine the expected number of de novo MEI mutations in exons, enhancers, and introns genome-wide. To calculate this value, we extrapolated $\mu$ for 9738 births (i.e., number of probands in our study), resulting in an estimate of 677 de novo MEIs genome wide. We then simulated this number of variants 100 times genome-wide using bedtools random ${ }^{45}$ and annotated the resulting simulated variants identically to real variants reported in this study (e.g., as in Fig. 2a). The total number of expected variants in the three categories depicted in Fig. 4 were then averaged across all simulations to determine Poisson $\lambda$ of de novo variants under neutral mutation rate. These distributions were then statistically compared with the number of observed variants in DDD using the ppois function in R (Fig. 4).

Reporting summary. Further information on research design is available in the Nature Research Reporting Summary linked to this article.

\section{Data availability}

Sequencing, phenotype data, and variant calls for all data in this paper are accessible via the European Genome-phenome Archive (EGA) under study number

EGAS00001000775 [https://www.ebi.ac.uk/ega/studies/EGAS00001000775]. Within this study, WES files of all DDD families are provided as part of data set EGAD00001004390, and RT variant calls and WGS data of the two individuals with de novo PPG variants are provided as part of data set EGAD00001004586.

\section{Code availability}

We have deposited R code, source data files, and raw blots from PCRs used to generate main text and Supplementary figs. online at the following github repository [https:// github.com/eugenegardner/MEIPaper.git]. Source code and more information for the PPG discovery pipeline used in this paper is available online at github [https://github. com/eugenegardner/Retrogene.git].

Received: 28 November 2018; Accepted: 11 September 2019; Published online: 11 October 2019

\section{References}

1. Mills, R. E., Bennett, E. A., Iskow, R. C. \& Devine, S. E. Which transposable elements are active in the human genome? Trends Genet. 23, 183-191 (2007)

2. Zhang, Z., Harrison, P. M., Liu, Y. \& Gerstein, M. Millions of years of evolution preserved: a comprehensive catalog of the processed pseudogenes in the human genome. Genome Res. 13, 2541-2558 (2003).

3. Stewart, C. et al. A comprehensive map of mobile element insertion polymorphisms in humans. PLoS Genet. 7, e1002236 (2011).

4. Sudmant, P. H. et al. An integrated map of structural variation in 2,504 human genomes. Nature 526, 75-81 (2015).

5. Gardner, E. J. et al. The mobile element locator tool (MELT): population-scale mobile element discovery and biology. Genome Res. 27, 1916-1929 (2017)

6. Zhang, Y., Li, S., Abyzov, A. \& Gerstein, M. B. Landscape and variation of novel retroduplications in 26 human populations. PLoS Comput. Biol. 13, e1005567 (2017).

7. Hancks, D. C. \& Kazazian, H. H. Jr. Roles for retrotransposon insertions in human disease. Mob. DNA 7, 9 (2016).

8. Brandler, W. M. et al. Frequency and complexity of de novo structural mutation in autism. Am. J. Hum. Genet. 98, 667-679 (2016).

9. Brandler, W. M. et al. Paternally inherited cis-regulatory structural variants are associated with autism. Science 360, 327-331 (2018).

10. Werling, D. M. et al. An analytical framework for whole-genome sequence association studies and its implications for autism spectrum disorder. Nat. Genet. 50, 727-736 (2018).

11. Hehir-Kwa, J. Y. et al. A high-quality human reference panel reveals the complexity and distribution of genomic structural variants. Nat. Commun. 7, 12989 (2016)

12. Deciphering Developmental Disorders Study. Prevalence and architecture of de novo mutations in developmental disorders. Nature 542, 433-438 (2017).

13. Martin, H. C. et al. Quantifying the contribution of recessive coding variation to developmental disorders. Science 362, 1161-1164 (2018).

14. King, D. A. et al. Detection of structural mosaicism from targeted and wholegenome sequencing data. Genome Res. 27, 1704-1714 (2017).

15. Short, P. J. et al. De novo mutations in regulatory elements in neurodevelopmental disorders. Nature 555, 611-616 (2018).

16. Niemi, M. E. K. et al. Common genetic variants contribute to risk of rare severe neurodevelopmental disorders. Nature 562, 268-271 (2018).

17. Lord, J. et al. Pathogenicity and selective constraint on variation near splice sites. Genome Res. 29, 159-170 (2019)

18. Kaplanis, J. et al. Exome-wide assessment of the functional impact and pathogenicity of multinucleotide mutations. Genome Res. 29, 1047-1056 (2019).

19. Goncalves, I., Duret, L. \& Mouchiroud, D. Nature and structure of human genes that generate retropseudogenes. Genome Res. 10, 672-678 (2000).

20. GTEx Consortium. Human genomics. The Genotype-Tissue Expression (GTEx) pilot analysis: multitissue gene regulation in humans. Science 348, 648-660 (2015).

21. Huang da, W., Sherman, B. T. \& Lempicki, R. A. Systematic and integrative analysis of large gene lists using DAVID bioinformatics resources. Nat. Protoc. 4, 44-57 (2009).

22. Lek, M. et al. Analysis of protein-coding genetic variation in 60,706 humans Nature 536, 285 (2016).

23. Samocha, K. E. et al. A framework for the interpretation of de novo mutation in human disease. Nat. Genet. 46, 944-950 (2014).

24. Hormozdiari, F. et al. Rates and patterns of great ape retrotransposition. Proc. Natl Acad. Sci. USA 110, 13457-13462 (2013).

25. Zhang, Y., Romanish, M. T. \& Mager, D. L. Distributions of transposable elements reveal hazardous zones in mammalian introns. PLoS Comput. Biol. 7, e1002046 (2011).

26. MacArthur, D. G. et al. A systematic survey of loss-of-function variants in human protein-coding genes. Science 335, 823-828 (2012) 
27. Kubiak, M. R. \& Makalowska, I. Protein-coding genes' retrocopies and their functions. Viruses 9, 80 (2017).

28. Gilbert, N., Lutz, S., Morrish, T. A. \& Moran, J. V. Multiple fates of L1 retrotransposition intermediates in cultured human cells. Mol. Cell Biol. 25 , 7780-7795 (2005).

29. Jonsson, H. et al. Parental influence on human germline de novo mutations in 1,548 trios from Iceland. Nature 549, 519-522 (2017).

30. Li, M. et al. Integrative functional genomic analysis of human brain development and neuropsychiatric risks. Science 362, eaat7615 (2018).

31. Riecken, L. B. et al. Inhibition of RAS activation due to a homozygous ezrin variant in patients with profound intellectual disability. Hum. Mutat. 36, 270-278 (2015).

32. Choufani, S. et al. NSD1 mutations generate a genome-wide DNA methylation signature. Nat. Commun. 6, 10207 (2015).

33. Watterson, G. A. On the number of segregating sites in genetical models without recombination. Theor. Popul Biol. 7, 256-276 (1975).

34. Ewing, A. D. \& Kazazian, H. H. Jr. High-throughput sequencing reveals extensive variation in human-specific L1 content in individual human genomes. Genome Res. 20, 1262-1270 (2010).

35. Collins, R. L. et al. An open resource of structural variation for medical and population genetics. Preprint at: https://www.biorxiv.org/content/early/2019/ 03/14/578674.full.pdf [biorxiv.org] (2019).

36. Witherspoon, D. J. et al. Mobile element scanning (ME-Scan) identifies thousands of novel Alu insertions in diverse human populations. Genome Res. 23, 1170-1181 (2013).

37. Wimmer, K., Callens, T., Wernstedt, A. \& Messiaen, L. The NF1 gene contains hotspots for L1 endonuclease-dependent de novo insertion. PLoS Genet. 7, e1002371 (2011).

38. Jurka, J. Sequence patterns indicate an enzymatic involvement in integration of mammalian retroposons. Proc. Natl Acad. Sci. USA 94, 1872-1877 (1997).

39. Sultana, T. et al. The landscape of L1 retrotransposons in the human genome is shaped by pre-insertion sequence biases and post-insertion selection. Mol. Cell 74, 555-570.e7 (2019).

40. Aneichyk, T. et al. Dissecting the causal mechanism of X-linked dystoniaparkinsonism by integrating genome and transcriptome assembly. Cell $\mathbf{1 7 2}$ 897-909.e21 (2018).

41. Wright, C. F. et al. Genetic diagnosis of developmental disorders in the DDD study: a scalable analysis of genome-wide research data. Lancet $\mathbf{3 8 5}$, 1305-1314 (2015).

42. Li, H. \& Durbin, R. Fast and accurate long-read alignment with BurrowsWheeler transform. Bioinformatics 26, 589-595 (2010).

43. Danecek, P. et al. The variant call format and VCFtools. Bioinformatics $\mathbf{2 7}$ 2156-2158 (2011).

44. McLaren, W. et al. The ensembl variant effect predictor. Genome Biol. 17, 122 (2016).

45. Quinlan, A. R. BEDTools: the Swiss-army tool for genome feature analysis. Curr. Protoc. Bioinforma. 47, 11.12.1-11.12.34 (2014).

46. May, D. et al. Large-scale discovery of enhancers from human heart tissue. Nat. Genet. 44, 89-93 (2011).

47. Visel, A., Minovitsky, S., Dubchak, I. \& Pennacchio, L. A. VISTA Enhancer Browser-a database of tissue-specific human enhancers. Nucleic Acids Res. 35, D88-D92 (2007)

48. Siepel, A. et al. Evolutionarily conserved elements in vertebrate, insect, worm, and yeast genomes. Genome Res. 15, 1034-1050 (2005).

49. Chaisson, M. J. \& Tesler, G. Mapping single molecule sequencing reads using basic local alignment with successive refinement (BLASR): application and theory. BMC Bioinforma. 13, 238 (2012).

50. McKenna, A. et al. The genome analysis toolkit: a MapReduce framework for analyzing next-generation DNA sequencing data. Genome Res. 20, 1297-1303 (2010).

51. Untergasser, A. et al. Primer3-new capabilities and interfaces. Nucleic Acids Res. 40, e115 (2012).

52. Tyner, C. et al. The UCSC Genome Browser database: 2017 update. Nucleic Acids Res. 45, D626-D634 (2016).

53. Thorvaldsdottir, H., Robinson, J. T. \& Mesirov, J. P. Integrative Genomics Viewer (IGV): high-performance genomics data visualization and exploration. Brief. Bioinform. 14, 178-192 (2013).

54. 1000 Genomes Project Consortium. A global reference for human genetic variation. Nature 526, 68-74 (2015).

55. Smit, A. F. A., Hubley, R., Green, P. RepeatMasker Open-3.0. [http://www. repeatmasker.org/ [repeatmasker.org]] (1996-2010)

56. O'Leary, N. A. et al. Reference sequence (RefSeq) database at NCBI: current status, taxonomic expansion, and functional annotation. Nucleic Acids Res. 44, D733-D745 (2016)

57. Firth, H. V. et al. DECIPHER: database of chromosomal imbalance and phenotype in humans using ensembl resources. Am. J. Hum. Genet. 84, 524-533 (2009).
58. Wright, C. F. et al. Making new genetic diagnoses with old data: iterative reanalysis and reporting from genome-wide data in 1,133 families with developmental disorders. Genet Med. 20, 1216-1223 (2018).

59. Kersey, P. J. et al. Ensembl Genomes 2016: more genomes, more complexity. Nucleic Acids Res. 44, D574-D580 (2016).

\section{Acknowledgements}

The authors wish to thank the Wellcome Sanger Institute sequencing facility staff for their assistance in preparing samples and performing sequencing experiments, all members of the DDD study for providing valuable comments during data analysis and the paper preparation, and the DDD families-this work would not be possible without their confidence and support. We also thank Panayiotis Constantinou for helping to curate known MEI-associated cases and for annotation of affected parents as well as Hilary Martin for constructive comments during manuscript preparation. We also wish to acknowledge Jeffrey Barrett and Caroline Wright for their leadership of the DDD. The DDD study presents independent research commissioned by the Health Innovation Challenge Fund [grant number: HICF-1009-003], a parallel funding partnership between Wellcome and the Department of Health, and the Wellcome Sanger Institute [grant number: WT098051]. The views expressed in this publication are those of the author(s), and not necessarily those of Wellcome or the Department of Health. The study has UK Research Ethics Committee approval (10/H0305/83, granted by the Cambridge South REC, and GEN/284/12 granted by the Republic of Ireland REC). The research team acknowledges the support of the National Institute for Health Research, through the Comprehensive Clinical Research Network. This study makes use of DECIPHER [http://decipher.sanger.ac.uk]), which is funded by the Wellcome.

\section{Author contributions}

E.J.G. performed RT variant calling and annotation, PPG algorithm design, constraint and burden testing, computational analysis of mosaic variants, and initial clinical annotation and together with M.E.H. designed experiments, oversaw the study, and wrote the paper. E. P. designed and performed PCR experiments. G.G. curated and prepared DDD sequencing data. S.S.G. and H.I. designed and conducted molecular assays to assess mosaic MEIs. P.J.S. assisted in estimating genetic burden of deleterious MEIs in the human population. A.S. assisted with the design of the PPG discovery algorithm. P.D., K.E.S., E.J.G., and T.S. performed variant calling and quality control of SNVs. J.H. performed methylation analysis of the NSD1 variant patient. K.E.C., E.C., K.L. L., K.P., E.R., D.R.F., and H.V.F. prepared clinical assessments of patients and confirmation of molecular diagnoses as they relate to patient phenotype.

\section{Competing interests}

M.E.H. is a co-founder of, consultant to, and holds shares in, Congenica Ltd, a genetics diagnostic company. The remaining authors declare no competing interests.

\section{Additional information}

Supplementary information is available for this paper at https://doi.org/10.1038/s41467019-12520-y.

Correspondence and requests for materials should be addressed to M.E.H.

Peer review information Nature Communications thanks Michael Talkowski and the other, anonymous, reviewer(s) for their contribution to the peer review of this work. Peer reviewer reports are available.

Reprints and permission information is available at http://www.nature.com/reprints

Publisher's note Springer Nature remains neutral with regard to jurisdictional claims in published maps and institutional affiliations.

Open Access This article is licensed under a Creative Commons Attribution 4.0 International License, which permits use, sharing, adaptation, distribution and reproduction in any medium or format, as long as you give appropriate credit to the original author(s) and the source, provide a link to the Creative Commons license, and indicate if changes were made. The images or other third party material in this article are included in the article's Creative Commons license, unless indicated otherwise in a credit line to the material. If material is not included in the article's Creative Commons license and your intended use is not permitted by statutory regulation or exceeds the permitted use, you will need to obtain permission directly from the copyright holder. To view a copy of this license, visit http://creativecommons.org/ licenses/by/4.0/.

(c) The Author(s) 2019 